\title{
A Simulation Study Of The Impact Of Forecast Recovery For Control Charts Applied To ARMA Processes
}

John N. Dyer

Georgia Southern University, jdyer@georgiasouthern.edu

B. Michael Adams

University Of Alabama

Michael D. Conerly

University Of Alabama

Follow this and additional works at: http://digitalcommons.wayne.edu/jmasm

Part of the Applied Statistics Commons, Social and Behavioral Sciences Commons, and the Statistical Theory Commons

\section{Recommended Citation}

Dyer, John N.; Adams, B. Michael; and Conerly, Michael D. (2002) "A Simulation Study Of The Impact Of Forecast Recovery For Control Charts Applied To ARMA Processes," Journal of Modern Applied Statistical Methods: Vol. 1 : Iss. 2 , Article 43.

DOI: $10.22237 /$ jmasm/1036110480

Available at: http://digitalcommons.wayne.edu/jmasm/vol1/iss2/43

This Regular Article is brought to you for free and open access by the Open Access Journals at DigitalCommons@WayneState. It has been accepted for inclusion in Journal of Modern Applied Statistical Methods by an authorized editor of DigitalCommons@WayneState. 


\section{A Simulation Study Of The Impact Of Forecast Recovery For Control Charts Applied To ARMA Processes}

\author{
John N. Dyer \\ Dept. Of Information Systems \\ \& Logistics \\ Georgia Southern University
}

\author{
B. Michael Adams \\ Dept. Of Information Systems, \\ Management Science, \& Statistics \\ University Of Alabama
}

\author{
Michael D. Conerly \\ Dept. Of Information Systems, \\ Management Science, \& Statistics \\ University Of Alabama
}

Forecast-based schemes are often used to monitor autocorrelated processes, but the resulting forecast recovery has a significant effect on the performance of control charts. This article describes forecast recovery for autocorrelated processes, and the resulting simulation study is used to explain the performance of control charts applied to forecast errors.

Key words: Autocorrelation, Forecast Recovery, Box-Jenkins, Quality, Simulation, Statistical Process Control, Statistics.

\section{Introduction}

Many traditional control charts were developed under the assumption that the measurements resulting from the in-control process are independent and identically distributed (iid) random variables.

Recently, many advances in measurement technology and sampling frequency yield sample measures that are not independently distributed. Hence, an alternative to the traditional control charting approach is to utilize a forecast-based monitoring scheme, which involves identifying the proper time-series model characterizing the process, obtaining the appropriate Box-Jenkins one-step-ahead forecast of process observations,

John N. Dyer is an Assistant Professor of Decision Sciences. His area of specialty is statistical process control with an emphasis in autocorrelated processes and forecast-based control charting. He is a member of ASA, ASQ, and DSI. Email: jdyer@gasou.edu, Phone: 912.681.5223, Fax: 912.681.0710. B. Michael Adams is an Associate Professor of Statistics. His primary areas of research include quality control and statistical process control. He is a member of ASA and ASQ. Email:badams@cba.ua.edu Michael D. Conerly is a Professor of Statistics. His areas of research include quality control and statistical process control. He is a member of ASA and ASQ. Email: mconerly@cba.ua.edu. and then applying traditional control charts to forecast errors (Alwan \& Roberts, 1988; Wardell, Moskowitz, \& Plante, 1994; Lin \& Adams, 1996; Lu \& Reynolds, 1999a; Lu \& Reynolds, 1999b; Lu \& Reynolds, 2001). If the assumed time-series model is correct, the forecast errors are iid normal random variables. Hence, the errors perform in a manner predictable through traditional control charting techniques, enabling monitoring for detection of step-shifts in the process mean level.

One problematic characteristic of forecastbased monitoring schemes is the phenomenon of forecast recovery; that is, the process forecasts recover quickly from process disturbances. Hence, the resulting forecast errors also recover quickly. This article describes models for autocorrelated data and the impact of forecast recovery for three special cases of the general autoregressive moving average (ARMA) model, and investigates the impact of forecast recovery on the Individuals, Exponentially Weighted Moving Average (EWMA), and the Combined EWMA-Shewhart (CES) control charts applied to forecast errors resulting from the ARMA models. A description of the simulation study is also provided. Recommendations are provided that will enable the practitioner to more readily identify the most appropriate control chart for use in monitoring various ARMA processes.

\section{Methodology}

When control chart performance has been evaluated, the average run length (ARL) has 
typically been used to quantify performance of the chart. The ARL is defined as the average number of time periods until the control chart signals. When the process is in-control, this is the expected time until a false-alarm. When the process shifts out-of-control, the ARL measures the expected time to detect the shift. The desired chart is one that simultaneously provides large in-control ARLs and low out-of-control ARLs. An alternative performance criterion is the cumulative distribution function (CDF). The CDF measures the cumulative proportion or percent of signals given by the $i^{\text {th }}$ period following the shift. It should be noted that the CDF completely characterizes the run length distribution, while the ARL is only the mean. Additionally, the median run length (MRL) can be used in conjunction with the ARL and CDF since it is a better measure of central tendency for skewed distributions such as the run length distribution. The MRL is defined as the median $\left(50^{\text {th }}\right.$ percentile $)$ number of time periods until the control chart signals. The desired chart is one with a high probability of early detection of a shift. In most cases, a trade-off between obtaining a low out-of-control ARL and high probability of early detection results.

The impact of forecast error recovery on ARLs has been discussed (Adams, Woodall, \& Superville; 1994; Superville \& Adams, 1994), and the CDF technique has been recommended as a meaningful criterion for evaluating the performance of charts on forecast errors. In light of forecast recovery, both ARL and CDF performance for step-shifts in the process mean were evaluated (Lin \& Adams, 1996) on the Individuals chart, the exponentially weighted moving average (EWMA) chart, and the combined EWMA-Shewhart (CES), in regard to monitoring forecast errors arising from particular forecastbased monitoring schemes. It was found that the Individuals chart provides relatively high ARLs and CDFs, the EWMA provides low ARLs and CDFs, and theCES borrows the best properties from both charts, low ARLs and high CDFs. High (low) CDFs are defined as those exhibiting a high (low) probability of initial shift detection relative to competing control charts.

In this article, control chart performance results are based primarily on ARL and CDF measures, but the MRL is also provided for each chart. Standard error of the run length (SRL) measures were provided to summarize the variability of each chart's run length distribution, as well as to give the reader an idea of the accuracy of each ARL measure. Performance results of the traditional control charts applied to forecast errors resulting from various $\operatorname{ARMA}(1,1)$, $\mathrm{AR}(1)$, and MA(1) processes with a step shift of $c$ $=1 \sigma_{\varepsilon}$ are given in Table 5 .

Simulations of the performance of the Individuals, EWMA, CES control applied to the forecast errors arising from various $\operatorname{ARMA}(1,1)$, $\mathrm{AR}(1)$, and $\mathrm{MA}(1)$ processes in this article give some insight into the impact of forecast recovery on these traditional control charts. This insight will better enable the practitioner to choose the appropriate control chart for various ARMA processes. The control charts were designed to provide in-control ARLs of 300. The EWMA and CES control charts were designed to detect a shift of the magnitude of the sustained expected forecast error for each model. A thorough discussion of sustained forecast recovery and sustained expected forecast error is provided in the following subsections.

\section{Simulation Description}

The simulation programs were designed, compiled, and run in Microsoft FORTRAN PowerStation for Windows, Version 4.0, utilizing FORTRAN 90. The program for finding ARLs were also used to estimate the appropriate control limits through trial and error. The simulations conducted are as follows.

1. A series of $4,100 \operatorname{ARMA}(2,1)$ variates were generated by FORTRAN MSIMSL subroutine RNARM. These variates were the simulated observations, $Y_{i}$ 's, for each of the models investigated.

2. The first 100 observations were used to allow a burn-in period.

3. A step shift was induced in the simulated observations. The magnitudes of shift range from 0 to $3 \sigma_{\varepsilon}$ in increments of $1 \sigma_{\varepsilon}$.

4. The appropriate Box-Jenkins OSA forecast and OSA forecast errors were calculated.

5. The programmed control chart monitored the forecast errors. The run lengths for the specified shift size were recorded.

6. Steps 1 through 5 were repeated 10,000 times for each model and process shift. The run length for the control chart was recorded for 
each simulation repetition and the ARL was obtained based on 10,000 repetitions. For the CDF programs, the percentages of runs producing a signal within the first 300 observations following the shift were obtained.

One issue concerning the simulation should be addressed. Each program can be run to simulate a process in a zero state or steady state. Zero state provides for simulating a process from start-up, while steady state provides for simulating a process that has been running in an in-control state for some time. When simulating for control limits and Null case ARL, MRL, and CDF performance, the programs were run from zero state. When simulating the ARL, MRL, and CDF performance for a process that has experienced a shift, the programs were run from steady state.

\section{Models for Autocorrelated Data}

Two $\operatorname{ARMA}(p, q)$ models have been found to have application in statistical process control. The first model of interest is the $\operatorname{ARMA}(1,1)$. Wardell, Moskowitz, and Plante (1992) address the $\operatorname{ARMA}(1,1)$ model, as it is a reasonable fit to data for some manufacturing processes. The second model of interest is the $\operatorname{ARMA}(1,0)$, also known as the $\mathrm{AR}(1)$. Montgomery and Mastrangelo (1991) and Alwan and Roberts (1988) have addressed the importance of the AR(1) model in manufacturing processes. Atienga, Tang and Ang (1998) discussed a time series approach to detecting level shifts in $\mathrm{AR}(1)$ processes. Lastly, the $\operatorname{ARMA}(0,1)$, also known as the $\operatorname{MA}(1)$, is considered for the sake of completion of all possible first order ARMA(p, q) models. The next section briefly discusses process shifts associated with the various time-series models before description of the models.

\section{ARMA(1,1), AR(1), MA(1) Models \& Process Shifts}

In building an empirical model of an actual time-series process, the inclusion of both autoregressive and moving average terms sometimes leads to a more parsimonious model than could be achieved with either the pure autoregressive or pure moving average alone. This results in the mixed autoregressive-moving average. When both terms are mixed in first order, the resulting model is the $\operatorname{ARMA}(1,1)$. The model for an in-control $\operatorname{ARMA}(1,1), \operatorname{AR}(1)$, and $\operatorname{MA}(1)$ processes are given by Eq.s (1), (2), and (3) respectively,

$$
\begin{aligned}
& \mathrm{Y}_{\mathrm{t}}=\xi+\phi \mathrm{Y}_{\mathrm{t}-1}+\varepsilon_{\mathrm{t}}-\theta_{\varepsilon_{\mathrm{t}-1}} \\
& \mathrm{Y}_{\mathrm{t}}=\xi+\phi \mathrm{Y}_{\mathrm{t}-1}+\varepsilon_{\mathrm{t}} \\
& \mathrm{Y}_{\mathrm{t}}=\xi-\theta \varepsilon_{\mathrm{t}-1}+\varepsilon_{\mathrm{t}}
\end{aligned}
$$

where $\xi$ is a constant and the sequence of $\varepsilon_{\mathrm{t}}(t=$ $1,2, \ldots)$ values are independent $\mathrm{N}\left(0, \sigma_{\varepsilon}^{2}\right)$ random variables. The $\operatorname{ARMA}(1,1)$ process is stationary for $|\phi|<1$ and $|\theta|<1$, the $\operatorname{AR}(1)$ process is stationary for $|\phi|<1$, and the $\operatorname{MA}(1)$ process is stationary for all values of $\theta$.

Now, suppose a step shift of size $c$ occurs in any of the $\operatorname{ARMA}(1,1), \operatorname{AR}(1)$, or $\operatorname{MA}(1)$ processes between time periods $r-1$ and $r$, that is, the process mean suddenly changes from $\xi$ to $\xi+\mathrm{c}$ at observation $r$. The Box-Jenkins one-step-ahead (OSA) forecasts are defined by $\hat{\mathrm{Y}}_{\mathrm{t}}=\xi+\phi \mathrm{Y}_{\mathrm{t}-1-\theta \mathrm{e}_{\mathrm{t}-1}}$ for the $\operatorname{ARMA}(1,1)$ process, $\hat{\mathrm{Y}}_{\mathrm{t}}=\xi+\phi \mathrm{Y}_{\mathrm{t}-1}$ for the $\mathrm{AR}(1)$ process, and $\hat{\mathrm{Y}}_{\mathrm{t}}=\xi-\theta \mathrm{e}_{\mathrm{t}-1}$ for the MA(1) process.

The OSA forecast errors are calculated as $e_{\mathrm{t}}=\mathrm{Y}_{\mathrm{t}}-\hat{\mathrm{Y}}_{\mathrm{t}}$, for all processes. The expected OSA forecast errors for an $\operatorname{ARMA}(1,1)$ process can be described mathematically as

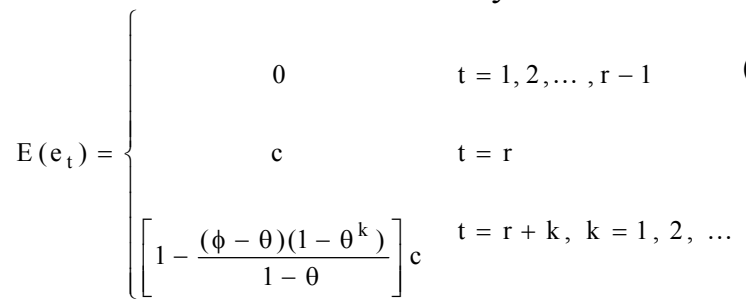

Similar results for the $\mathrm{AR}(1)$ and $\mathrm{MA}(1)$ processes can be obtained by setting $\theta=0$ or $\phi=$ 0 , respectively in Eq. (4). This general representation is consistent with the special cases of the ARMA $(1,1)$ model presented in Atienga, Tang and Ang (1998), Lin and Adams (1996), and Wardell, Moskowitz and Plante (1994).

Tables 1,2 , and 3 portray a realization of the expectation of forecast errors at time periods $t$ $<r, t=r$, and $t>r$, for a $c=1 \sigma_{\varepsilon}$ step shift in $\operatorname{ARMA}(1,1), \operatorname{AR}(1)$, and MA(1) models. These choices of models were designed by Wardell, Moskowitz, and Plante (1994) to systematically cover the region over which the ARMA series is 
stationary. Although the models possessing positive autocorrelation are most likely to be encountered in manufacturing processes, those possessing negative correlation may be more prevalent in nonmanufacturing applications.

When an $\operatorname{ARMA}(p, q)$ process undergoes a step shift in the mean, the expected value of the forecast of the process varies for a time and then converges to a new equilibrium level (Wardell et al. (1994)), referred to in this paper as the sustained level of the shift. The response of the forecasts also causes the forecast errors to respond dynamically, as can be seen in Tables 1, 2, and 3 . For the $\operatorname{ARMA}(1,1)$ model, the forecast errors react much differently, depending on the degree and direction of the first order autocorrelation, $\rho_{1}$, as well as the values of $\phi_{1}$ and $\theta_{1}$. For all $\operatorname{ARMA}(\mathrm{p}, \mathrm{q})$ models, the expected forecast error at time $t=r$ is equal to $c$, but the dynamic response of the errors can vary dramatically for times $t>r$.

Table 1: Forecast Error Expectation for Positively Autocorrelated ARMA(1,1) Processes with a Shift of $c$ $=1 \sigma_{\varepsilon}$ at Time Period $t=r$.

\begin{tabular}{ccccccccc}
\hline Model & $\mathbf{1}$ & $\mathbf{2}$ & $\mathbf{3}$ & $\mathbf{4}$ & $\mathbf{5}$ & $\mathbf{6}$ & $\mathbf{7}$ & $\mathbf{8}$ \\
\hline$\phi_{1}$ & .950 & .950 & .950 & .950 & .475 & .475 & .475 & -.475 \\
$\theta_{1}$ & .900 & .450 & -.45 & -.90 & .450 & -.45 & -.90 & -.900 \\
$\rho_{1}$ & .072 & .824 & .971 & .975 & .025 & .689 & .737 & .255 \\
\hline $\mathbf{t}$ & \multicolumn{7}{c}{ Expected Forecast Errors, E( $\left.\boldsymbol{e}_{\boldsymbol{t}}\right)$} \\
\hline$<\mathrm{r}$ & .00 & 0.00 & 0.00 & 0.00 & 0.00 & 0.00 & 0.00 & 0.00 \\
$\mathrm{r}$ & 1.0 & 1.00 & 1.00 & 1.00 & 1.00 & 1.00 & 1.00 & 1.00 \\
$\mathrm{R}+1$ & .95 & 0.50 & -.40 & -.85 & 0.98 & 0.08 & -.38 & 0.58 \\
$\mathrm{R}+2$ & .91 & 0.28 & 0.23 & 0.82 & 0.96 & 0.49 & 0.86 & 0.96 \\
$\mathrm{R}+3$ & .86 & 0.17 & -.05 & -.68 & 0.96 & 0.30 & -.25 & 0.61 \\
$\mathrm{R}+4$ & .83 & 0.13 & 0.07 & 0.67 & 0.96 & 0.39 & 0.75 & 0.92 \\
$\mathrm{R}+5$ & .80 & 0.11 & 0.02 & -.55 & 0.96 & 0.35 & -.15 & 0.64 \\
. &. &. &. &. &. &. &. &. \\
$\mathrm{r}+44$ & .50 & 0.09 & 0.03 & 0.04 & 0.95 & 0.36 & 0.28 & 0.78 \\
$\mathrm{r}+45$ & .50 & 0.09 & 0.03 & 0.02 & 0.95 & 0.36 & 0.27 & 0.77 \\
\hline
\end{tabular}

For positively autocorrelated $\operatorname{ARMA}(1,1)$ processes, the following is observed in Table 1: The $\mathrm{E}\left(e_{\mathrm{t}}\right)$ recovers to a value less than $c$ for all times $t>r$. The recovery rate depends not only upon the values of $\phi_{1}$ and $\theta_{1}$, but also upon the particular time $t$ after the shift. Defining $\mathrm{E}\left(e_{t}{ }^{*}\right)$ to be the expected sustained level of the original shift of size $c$ resulting from an $\operatorname{ARMA}(1,1)$ process, Eq. (5) can be derived from Eq. (4) when $t>r$, as $k \rightarrow \infty$, and it can be shown that
Table 2: Forecast Error Expectation for Negatively Autocorrelated ARMA $(1,1)$ Processes with a Shift of c $=1 \sigma_{\varepsilon}$ at Time Period $t=r$.

\begin{tabular}{|c|c|c|c|c|c|c|c|c|}
\hline$\phi_{1}$ & .475 & -.475 & $\begin{array}{c}- \\
.475\end{array}$ &. & -.950 & -.95 & -.95 & -.95 \\
\hline$\theta_{1}$ & .900 & .900 & .450 & -.45 & .900 & .450 & -.45 & -.90 \\
\hline$\rho_{1}$ & -.255 & -.737 & $\begin{array}{c}- \\
.689\end{array}$ & .025 & -.975 & .971 &. & $\begin{array}{c}- \\
.072\end{array}$ \\
\hline $\mathbf{t}$ & \multicolumn{8}{|c|}{ Expected Forecast Errors, E $\left(e_{t}\right)$} \\
\hline$<\mathrm{r}$ & 0.00 & 0.0 & 0.00 & 0.00 & 0.00 & 0.00 & 0.00 & 0.00 \\
\hline $\mathrm{r}$ & 1.00 & 1.0 & 1.00 & 1.00 & 1.00 & 1.00 & 1.00 & 1.00 \\
\hline$r+1$ & 1.43 & 2.38 & 1.93 & 1.03 & 2.85 & 2.40 & 1.50 & 1.05 \\
\hline$r+2$ & 1.81 & 3.61 & 2.34 & 1.01 & 4.52 & 3.03 & 1.28 & 1.01 \\
\hline$r+3$ & 2.15 & 4.73 & 2.53 & 1.02 & 6.01 & 3.31 & 1.38 & 1.05 \\
\hline$r+4$ & 2.46 & 5.73 & 2.61 & 1.02 & 7.36 & 3.44 & 1.33 & 1.01 \\
\hline$r+5$ & 2.74 & 6.63 & 2.65 & 1.02 & 8.58 & 3.50 & 1.35 & 1.04 \\
\hline . & . & & . & . & & . & . & • \\
\hline$r+44$ & 5.21 & 14.62 & 2.68 & 1.02 & 19.32 & 3.55 & 1.34 & 1.03 \\
\hline$r+45$ & 5.21 & 14.63 & 2.68 & 1.02 & 19.34 & 3.55 & 1.34 & 1.03 \\
\hline
\end{tabular}

Table 3: Forecast Error Expectation for AR (1) and MA(1) Processes with a Shift of $c=1 \sigma_{\varepsilon}$ at Time Period $t=r$

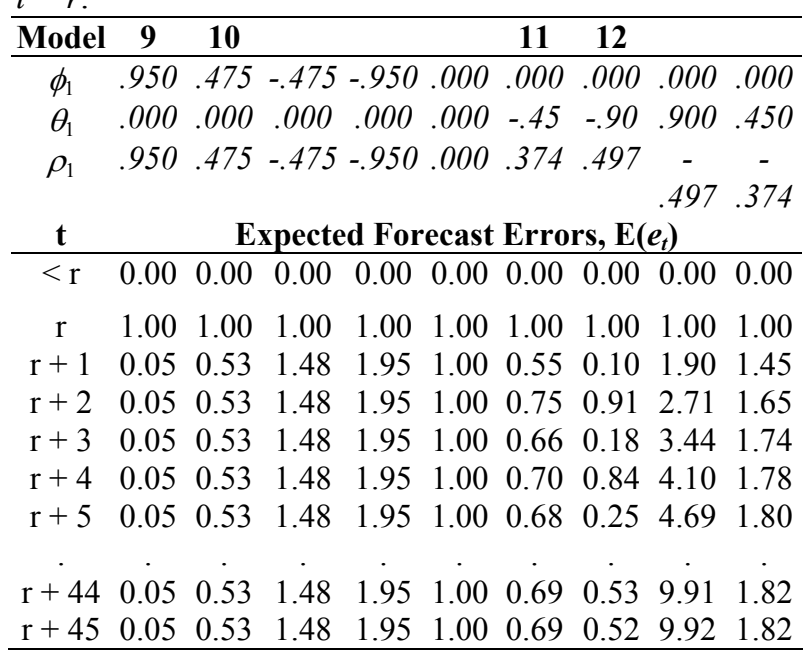

$$
\mathrm{E}\left(e_{\mathrm{t}}\right) \rightarrow\left[1-\frac{\left(\phi_{1}-\theta_{1}\right)}{\left(1-\theta_{1}\right)}\right] c=\mathrm{E}\left(e_{t}^{*}\right) .
$$

Table 4 contains values of $\mathrm{E}\left(e_{t}^{*}\right)$ for various combinations of $\phi_{1}$ and $\theta_{1}$, hence providing a realization of the dynamic response of the forecast errors. Again, the degree of autocorrelation as well as the values of $\phi_{1}$ and $\theta_{1}$ determines the rate of convergence. It is obvious from Eq. (4) that $k$ enters into the determination of $\mathrm{E}\left(e_{t}\right)$ only through $\theta_{1}$; hence, only $\operatorname{ARMA}(1,1)$ and $\operatorname{MA}(1)$ models with nonzero $\theta_{1}$ converge to $\mathrm{E}\left(e_{t}^{*}\right)$. In general, it 
appears that $\operatorname{ARMA}(1,1)$ models with large $\left|\theta_{1}\right|$ converge more slowly than when $\left|\theta_{1}\right|$ is small.

Models with large $\rho_{1}$ tend to converge to a value of $\mathrm{E}\left(e_{t}^{*}\right)$ close to zero, while models with small $\rho_{1}$ (i.e., close to zero) tend to quickly attain a value of $\mathrm{E}\left(e_{t}{ }^{*}\right)$ close to $c$. For some combinations of $\phi_{1}$ and $\theta_{1}$, most noticeably $\phi_{1}$ positive while $\theta_{1}$ negative, the $\mathrm{E}\left(e_{t}\right)$ oscillates between values less than $c$, until finally converging to $\mathrm{E}\left(e_{t}^{*}\right)$. Again, depending upon the magnitude of $\phi_{1}$ and $\theta_{1}$, the oscillation may go between positive and negative values less than $c\left(\phi_{1}=0.95, \theta_{1}=-0.45\right)$, or between strictly positive values less than $c\left(\phi_{1}=\right.$ $\left.0.475, \theta_{1}=-0.45\right)$.

For negatively autocorrelated $\operatorname{ARMA}(1,1)$ processes, Table 2 reveals that the $\mathrm{E}\left(e_{t}\right)$ exceeds $c$ for all times $t>r$. The magnitude of $\mathrm{E}\left(e_{t}\right)$ again depends on the values of $\phi_{1}$ and $\theta_{1}$, as well as the time $t$ following the shift. In most instances where $\rho_{1}$ approaches zero, $\mathrm{E}\left(e_{t}{ }^{*}\right)$ assumes a value trivially larger than $c$. In instances where $\rho_{1}$ approaches negative one, $\mathrm{E}\left(e_{t}{ }^{*}\right)$ often assumes a value much larger than $c$. Again, some oscillation among the values of $\mathrm{E}\left(e_{t}\right)$ occurs at times $t>r$, but not to the degree as when $\rho_{1}$ is positive. Only $\operatorname{ARMA}(1,1)$ processes exhibiting forecast recovery, that is, positively autocorrelated processes, are further considered in this article. The $\operatorname{ARMA}(1,1)$ processes in Table 1 to be further considered are labeled Models 1 through 8.

For positively autocorrelated $\mathrm{AR}(1)$ processes, the following is observed in Table 3: $\mathrm{E}\left(e_{t}\right)$ recovers to a constant value less than $c$ for all $t>r$, for all $\rho_{1}$ between zero and one. Larger values of $\phi_{1}$ lead to greater degrees of forecast recovery. Defining $\mathrm{E}\left(e_{t}^{*}\right)$ to be the expected sustained level of the original shift of size $c$ resulting from an AR(1) process, Eq. (6) can be derived from Eq. (4) for all periods $t>r$, and it can be shown that

$$
\mathrm{E}\left(e_{t}^{*}\right)=\left(1-\phi_{1}\right) c .
$$

For negatively autocorrelated AR(1) processes, the following is observed in Table 3: $\mathrm{E}\left(e_{t}\right)$ increases to a constant value greater than $c$ for all $t>r$, for all $\rho_{1}$ between zero and negative one. Values of $\phi_{1}$ closer to negative one lead to greater increases in values of the expected forecast errors. Only AR(1) processes exhibiting forecast recovery, that is, positively autocorrelated processes, are considered in this article. The AR(1) processes to be further considered in Table 3 are labeled Models 9 and 10.

For positively autocorrelated MA(1) processes, the following is observed in Table 3: $\mathrm{E}\left(e_{t}\right)$ recovers to a value less than $c$ for all times $t$ $>r$. The recovery rate depends not only upon the value of $\theta_{1}$, but upon the particular time $t$ after the shift. Defining $\mathrm{E}\left(e_{t}^{*}\right)$ to be the expected sustained level of the original shift of size $c$ resulting from an MA(1) process, Eq. (7) can be derived from Eq. (4) when $t>r$, as $k \rightarrow \infty$, and it can be shown that

$$
\mathrm{E}(e \mathrm{t}) \rightarrow\left[\frac{1}{\left(1-\theta_{1}\right)}\right] c=\mathrm{E}\left(e_{t}^{*}\right) .
$$

The degree of autocorrelation as well as the value of $\theta_{1}$ determines the rate of convergence. As in the case with the $\operatorname{ARMA}(1,1), \mathrm{E}\left(e_{t}\right)$ oscillates, converging to the value $\mathrm{E}\left(e_{t}^{*}\right)$, which is less than $c$, for all $t>r$. At no time does $\mathrm{E}\left(e_{t}\right)$ exceed the value $c$. For negatively autocorrelated MA(1) processes, the following holds: the $\mathrm{E}\left(e_{t}\right)$ exceeds $c$ for all times $t>r$. The magnitude of $\mathrm{E}\left(e_{t}\right)$ again depends on the value $\theta_{1}$, as well as the time $t$ following the shift. The response of $\mathrm{E}\left(e_{t}\right)$ and the sustained level of the shift, $\mathrm{E}\left(e_{t}^{*}\right)$, is much like that for the $\operatorname{ARMA}(1,1)$ model in regards to various degrees of autocorrelation. Only MA(1) processes exhibiting forecast recovery, that is, positively autocorrelated processes, are considered in this article. The MA(1) processes to be considered in Table 3 are labeled Models 11 and 12 .

Table 4 contains the sustained expected forecast error values, $\mathrm{E}\left(e_{t}^{*}\right)$, for various combinations of $\phi_{1}$ (left most column) and $\theta_{1}$ (top most row) for $\operatorname{ARMA}(1,1), \operatorname{AR}(1)$, and $\operatorname{MA}(1)$ models, given a $c=1 \sigma_{\varepsilon}$ shift in the process mean level. The values $\phi_{1}$ and $\theta_{1}$ corresponding to the upper diagonal of Table 4 produce values of $\mathrm{E}\left(e_{t}\right)$ whose sustained level of shift is less than $c$. In this case, the forecast errors are said to recover. The lower diagonal region contains values of $\mathrm{E}\left(e_{t}\right)$, whose sustained level of shift is greater than or equal to $c$. All entries represent combinations of $\phi_{1}$ and $\theta_{1}$ that result in stationary $\operatorname{ARMA}(1,1)$ processes. 
Now consider the following example for understanding Table 4. Given an $\operatorname{ARMA}(1,1)$ model $\left(\phi_{1}=-0.15, \theta_{1}=-0.65\right)$ with time $t<r$ (incontrol) $\mathrm{E}\left(e_{t}\right)$ of zero, the values of $\mathrm{E}\left(e_{t}\right)$ at times $t$ $<r, t=r$, and $t>r$, are as follows for a $c=1 \sigma_{\varepsilon}$ shift in the process mean:

$$
E\left(e_{t}\right)=\left\{\begin{array}{cl}
0 & t=1,2, \ldots r-1 \\
1.00 & t=r \\
{\left[1-\frac{(-0.15+0.65)}{(1+0.65)}\right]=0.70} & t=r+k \quad k \rightarrow \infty .
\end{array}\right.
$$

Notice that $\mathrm{E}\left(e_{t}\right)$ in Eq. (8) at time $t>r$ is equal to 0.70 for $k \rightarrow \infty$. The intersection of $\phi_{1}=-$ 0.15 (left most column) and $\theta_{1}=-0.65$ (top most row) in Table 4 also yields the expected forecast error value of 0.70 . Additionally, the relationship between the lag one autocorrelation, $\rho_{1}$, for the various combinations of $\phi_{1}$ and $\theta_{1}$, and the sustained expected forecast errors, $\mathrm{E}\left(e_{t}^{*}\right)$, in Table 4 , is very strong and linear, with a correlation of $r$ $=-0.997$. Eq. (9) provides an estimate of the sustained expected forecast errors as a function of $\rho_{1}$ for most $\operatorname{ARMA}(1,1), \operatorname{AR}(1)$, or MA(1) models. Again, it is obvious from this relationship that large first order autocorrelation provides for more extreme forecast recovery.

$$
\hat{E}\left(e_{t}^{*}\right)=1.04-1.02 \rho_{1} .
$$

Considering the AR parameter alone, forecast recovery occurs for all values of $\phi_{1}>0$, while the most extreme sustained forecast recovery occurs for values of $\phi_{1}>0.50$. Considering the MA parameter alone, the sustained level of forecast error recovery never falls below $\mathrm{E}\left(e_{t}^{*}\right)=0.50$, so no value of $\theta_{1}$ alone results in extreme forecast recovery. Considering both parameters, the most extreme sustained forecast recovery occurs when $\phi_{1}>0$ while $\theta_{1}<0$, and in most cases in which $\phi_{1}$ is large, that is, $\phi_{1}>$ 0.50 , regardless of the value of $\theta_{1}$.

\section{Results}

Recall that the degree and rate of forecast recovery, as well as the time until sustained level of forecast recovery occurs provide a source of conflict when choosing among control charts for monitoring forecast errors. Traditionally, if the ARL is used for the basis of comparison, the EWMA control chart most often provides smaller out-of-control ARLs than any other chart for small shifts, particularly when compared to the Individuals chart. However, the Individuals chart generally provides the greatest probability of obtaining a signal within the first few observations following the shift although a much larger ARL is provided. One can best understand the impact of forecast recovery by first examining chart performance applied to the AR(1) processes.

\section{Control Charts Applied to AR(1) Models}

Recall that when a shift occurs in any AR(1) process, the first forecast error following the shift appreciates the full impact of the shift, $c$. The forecast errors suddenly recover for all subsequent periods to a sustained level less than the original shift, $\left(1-\phi_{1}\right) c$. In contrast, the $\operatorname{ARMA}(1,1)$ and $\operatorname{MA}(1)$ processes recover gradually over time until finally converging to the sustained level less than the original shift. Depending on the particular process, oscillation may occur between values of sequential forecast errors. Since the forecast errors arising from the $\mathrm{AR}(1)$ process recover instantly to the sustained level of the shift, the worst performance of most control charts applied to a general $\operatorname{ARMA}(p, q)$ process should usually be obtained in the case of the AR(1) process for a given shift and sustained level of the shift. Performance results of the traditional control charts applied to forecast errors resulting from various $\operatorname{ARMA}(1,1), \operatorname{AR}(1)$, and MA(1) processes with a step shift of $c=1 \sigma_{\varepsilon}$ are given in Table 5.

Table 5, Models 9 and 10, show that the EWMA control chart maintains good ARL performance relative to the Individuals chart over a wide range of $\mathrm{AR}(1)$ parameter values and shift sizes, but the Individuals control chart consistently provides higher probabilities of initial shift detection, particularly for larger shifts (not shown). As found by Lin and Adams (1996), the CES control chart provides out-of-control ARLs similar to those of the EWMA chart while simultaneously maintaining the high probability of an early signal provided by the Individuals chart. 


\begin{tabular}{|c|c|c|c|c|c|c|c|c|c|c|c|c|c|c|c|c|c|c|c|c|c|}
\hline & -.95 & -.85 & -.75 & -.65 & -.55 & -.45 & -.35 & -.25 & -.15 & -.05 & .00 & .05 & .15 & .25 & .35 & .45 & .55 & .65 & .75 & .85 & .95 \\
\hline .95 & .03 & .03 & .03 & .03 & .03 & .03 & .04 & .04 & .04 & .05 & .05 & .05 & .06 & .07 & .08 & .09 & .11 & .14 & .20 & .33 & 1.0 \\
\hline .85 & .08 & .08 & .09 & .09 & 10. & 10 & 11 & .12. & .13 & .14 & .15 & .16 & .18 & .20 & .23 & .27 & .33 & .43 & .60 & 1.0 & 3.0 \\
\hline .75 & .13 & 14 & .14 & .15 & .16 & .17 & 19 & .20 & .22 & .24 & .25 & .26 & .29 & .33 & .38 & .45 & .56 & .71 & 1.0 & 1.7 & 5.0 \\
\hline .65 & .18 & 19 & .20 & .21 & .23 & .24 & .26 & .28 & .30 & .33 & .35 & .37 & .41 & .47 & .54 & .64 & .78 & 1.0 & 1.4 & 2.3 & 7.0 \\
\hline .55 & .23 & .24 & .26 & .27 & .29 & .31 & .33 & .36 & .39 & .43 & .45 & .47 & .53 & .60 & .69 & .82 & 1.0 & 1.3 & 1.8 & 3.0 & 9.0 \\
\hline .45 & .28 & .30 & .31 & .33 & .35 & .38 & .41 & .44 & .48 & .52 & .55 & .58 & .65 & .73 & .85 & 1.0 & 1.2 & 1.6 & 2.2 & 3.7 & 11 \\
\hline .35 & .33 & .35 & .37 & .39 & .42 & .45 & .48 & .52 & .57 & .62 & .65 & .68 & .76 & .87 & 1.0 & 1.2 & 1.4 & 1.9 & 2.6 & 4.3 & 13 \\
\hline .25 & .38 & .41 & .43 & .45 & .48 & .52 & .56 & .60 & .65 & .71 & .75 & .79 & .88 & 1.0 & 1.2 & 1.4 & 1.7 & 2.1 & 3.0 & 5.0 & 15 \\
\hline .15 & .44 & .46 & .49 & .52 & .55 & .59 & .63 & .68 & .74 & .81 & .85 & .89 & 1.0 & 1.1 & 1.3 & 1.5 & 1.9 & 2.4 & 3.4 & 5.7 & 17 \\
\hline .05 & .49 & .51 & .54 & .58 & .61 & .66 & .70 & .76 & .83 & .90 & .95 & 1.0 & 1.1 & 1.3 & 1.5 & 1.7 & 2.1 & 2.7 & 3.8 & 6.3 & 19 \\
\hline .00 & .51 & .54 & .57 & .61 & .65 & .69 & .74 & .80 & .87 & .95 & 1.0 & 1.1 & 1.2 & 1.3 & 1.5 & 1.8 & 2.2 & 2.9 & 4.0 & 6.7 & 20 \\
\hline-.05 & .54 & .57 & .60 & .64 & .68 & .72 & .78 & .84 & .91 & 1.0 & 1.1 & 1.1 & 1.2 & 1.4 & 1.6 & 1.9 & 2.3 & 3.0 & 4.2 & 7.0 & 21 \\
\hline -15 & .59 & .62 & .66 & .70 & .74 & .79 & .85 & .92 & 1.0 & 1.1 & 1.2 & 1.2 & 1.4 & 1.5 & 1.8 & 2.1 & 2.6 & 3.3 & 4.6 & 7.7 & 23 \\
\hline-.25 & .64 & .68 & .71 & .76 & .81 & .86 & .93 & 1.0 & 1.1 & 1.2 & 1.3 & 1.3 & 1.5 & 1.7 & 1.9 & 2.3 & 2.8 & 3.6 & 5.0 & 8.3 & 25 \\
\hline-.35 & .69 & .73 & .77 & .82 & .87 & .93 & 1.0 & 1.1 & 1.2 & 1.3 & 1.4 & 1.4 & 1.6 & 1.8 & 2.1 & 2.5 & 3.0 & 3.9 & 5.4 & 9.0 & 27 \\
\hline-.45 & .74 & .78 & .83 & .88 & .94 & 1.0 & 1.1 & 1.2 & 1.3 & 1.4 & 1.5 & 1.5 & 1.7 & 1.9 & 2.2 & 2.6 & 3.2 & 4.1 & 5.8 & 9.7 & 29 \\
\hline-.55 & .79 & .84 & .89 & .94 & 1.0 & 1.1 & 1.1 & 1.2 & 1.3 & 1.5 & 1.6 & 1.6 & 1.8 & 2.1 & 2.4 & 2.8 & 3.4 & 4.4 & 6.2 & 10 & 31 \\
\hline-.65 & .85 & .89 & .94 & 1.0 & 1.1 & 1.1 & 1.2 & 1.3 & 1.4 & 1.6 & 1.7 & 1.7 & 1.9 & 2.2 & 2.5 & 3.0 & 3.7 & 4.7 & 6.6 & 11 & 33 \\
\hline-.75 & .90 & .95 & 1.0 & 1.1 & 1.1 & 1.2 & 1.3 & 1.4 & 1.5 & 1.7 & 1.8 & 1.8 & 2.1 & 2.3 & 2.7 & 3.2 & 3.9 & 5.0 & 7.0 & 12 & 35 \\
\hline-85 & .95 & 1.0 & 1.1 & 1.1 & 1.2 & 1.3 & 1.4 & 1.5 & 1.6 & 1.8 & 1.9 & 1.9 & 2.2 & 2.5 & 2.8 & 3.4 & 4.1 & 5.3 & 7.4 & 12 & 37 \\
\hline-.95 & 1.0 & 1.1 & 1.1 & 1.2 & 1.3 & 1.3 & 1.4 & 1.6 & 1.7 & 1.9 & 2.0 & 2.1 & 2.3 & 2.6 & 3.0 & 3.5 & 4.3 & 5.6 & 7.8 & 13 & 39 \\
\hline
\end{tabular}

Table 4: Sustained Expected Forecast Errors for Combinations of $\phi_{1}$ and $\theta_{1}$.

As the degree of forecast recovery worsens though, ARL and CDF performance decreases for all of the control charts. In regard to these traditional control charts, the CES chart provides the best compromising performance over a wide range of $\mathrm{AR}(1)$ process parameter values and shift sizes.
Performance of Control Charts Applied to $\operatorname{ARMA}(1,1)$ and MA(1) Models

For the $\operatorname{ARMA}(1,1)$ and MA(1) processes, the behavior of the forecast errors prior to the sustained level has an impact on all of the control charts. 
Table 5: ARLs , MRLs, and CDFs for the ARMA(1,1) Process with Step Shift $c=1 \sigma_{\varepsilon}$.

\begin{tabular}{|c|c|c|c|c|c|c|c|c|c|c|c|}
\hline \multirow{2}{*}{$\begin{array}{l}\text { ARMA } \\
\text { Model }\end{array}$} & \multirow{2}{*}{$\begin{array}{c}\text { Control } \\
\text { Chart }\end{array}$} & \multirow[b]{2}{*}{ ARL } & \multirow[b]{2}{*}{ MRL } & \multirow[b]{2}{*}{ SRL } & \multicolumn{7}{|c|}{ Cumulative Percentage of Signals Following Shift } \\
\hline & & & & & $1^{\text {st }}$ & $2^{\text {nd }}$ & $3^{\text {rd }}$ & $4^{\text {th }}$ & $5^{\text {th }}$ & $6^{\text {th }}$ & $7^{\text {th }}$ \\
\hline \multirow{3}{*}{1} & IND & 115 & 70 & 128 & 2.52 & 4.67 & 6.81 & 8.61 & 10.06 & 11.70 & 13.10 \\
\hline & EWMA & 15 & 12 & 12 & 2.03 & 4.04 & 6.95 & 10.75 & 15.48 & 21.03 & 26.94 \\
\hline & CES & 21 & 16 & 19 & 2.41 & 4.65 & 7.05 & 9.55 & 12.28 & 15.48 & 18.91 \\
\hline \multirow{3}{*}{2} & IND & 279 & 191 & 284 & 2.52 & 3.23 & 3.62 & 3.98 & 4.28 & 4.62 & 5.00 \\
\hline & EWMA & 136 & 96 & 141 & 7.15 & 8.32 & 9.35 & 10.05 & 10.74 & 11.31 & 11.91 \\
\hline & CES & 184 & 136 & 177 & 3.03 & 3.88 & 4.41 & 4.86 & 5.27 & 5.66 & 6.13 \\
\hline \multirow{3}{*}{3} & IND & 290 & 199 & 295 & 2.52 & 3.23 & 3.60 & 3.93 & 4.23 & 4.53 & 4.88 \\
\hline & EWMA & 217 & 145 & 239 & 7.01 & 7.92 & 8.63 & 9.22 & 9.83 & 10.39 & 10.86 \\
\hline & CES & 259 & 178 & 265 & 3.03 & 3.74 & 4.19 & 4.54 & 4.90 & 5.24 & 5.64 \\
\hline \multirow{3}{*}{4} & IND & 270 & 177 & 293 & 2.52 & 4.34 & 5.97 & 7.14 & 8.10 & 8.93 & 9.75 \\
\hline & EWMA & 227 & 150 & 252 & 7.03 & 8.16 & 8.96 & 9.48 & 10.16 & 10.71 & 11.22 \\
\hline & CES & 252 & 165 & 276 & 3.03 & 4.77 & 6.32 & 7.39 & 8.32 & 9.08 & 9.86 \\
\hline \multirow{3}{*}{5} & IND & 42 & 29 & 42 & 2.52 & 4.80 & 7.25 & 9.44 & 11.43 & 13.63 & 15.80 \\
\hline & EWMA & 9 & 8 & 5 & 1.51 & 4.28 & 8.73 & 15.38 & 23.33 & 32.56 & 41.75 \\
\hline & CES & 12 & 11 & 7 & 2.34 & 4.93 & 8.09 & 12.14 & 16.89 & 22.85 & 29.38 \\
\hline \multirow{3}{*}{6} & IND & 177 & 122 & 180 & 2.52 & 2.94 & 3.68 & 4.13 & 4.59 & 5.10 & 5.67 \\
\hline & EWMA & 35 & 30 & 26 & 2.59 & 3.38 & 4.34 & 5.13 & 6.09 & 7.28 & 8.46 \\
\hline & CES & 51 & 43 & 39 & 2.45 & 2.95 & 3.71 & 4.30 & 4.95 & 5.68 & 6.53 \\
\hline \multirow{3}{*}{7} & IND & 205 & 138 & 218 & 2.52 & 3.18 & 5.08 & 5.46 & 6.70 & 7.03 & 8.15 \\
\hline & EWMA & 48 & 41 & 37 & 4.46 & 5.19 & 6.27 & 6.72 & 7.80 & 8.31 & 9.32 \\
\hline & CES & 67 & 57 & 52 & 2.79 & 3.48 & 5.34 & 5.76 & 6.97 & 7.33 & 8.53 \\
\hline \multirow{3}{*}{8} & IND & 64 & 43 & 63 & 2.52 & 3.38 & 5.84 & 6.85 & 8.78 & 9.92 & 11.85 \\
\hline & EWMA & 13 & 11 & 8 & 1.61 & 3.06 & 5.99 & 9.03 & 14.30 & 19.20 & 26.22 \\
\hline & CES & 17 & 15 & 11 & 2.36 & 3.40 & 6.11 & 7.77 & 11.08 & 13.76 & 18.36 \\
\hline $\mathrm{AR}(1)$ & IND & 290 & 199 & 295 & 2.52 & 2.93 & 3.24 & 3.56 & 3.84 & 4.14 & 4.51 \\
\hline \multirow[t]{2}{*}{9} & EWMA & 194 & 131 & 209 & 7.05 & 7.95 & 8.72 & 9.26 & 9.89 & 10.44 & 10.97 \\
\hline & CES & 242 & 167 & 246 & 3.03 & 3.51 & 3.93 & 4.30 & 4.66 & 5.03 & 5.44 \\
\hline $\mathrm{AR}(1)$ & IND & 119 & 81 & 120 & 2.52 & 3.27 & 4.08 & 4.91 & 5.60 & 6.41 & 7.21 \\
\hline \multirow[t]{2}{*}{10} & EWMA & 21 & 19 & 14 & 2.24 & 3.51 & 4.82 & 6.24 & 8.10 & 10.16 & 12.67 \\
\hline & CES & 29 & 26 & 20 & 2.44 & 3.28 & 4.18 & 5.22 & 6.32 & 7.59 & 8.98 \\
\hline $\mathrm{MA}(1)$ & IND & 79 & 54 & 79 & 2.52 & 3.34 & 4.77 & 5.93 & 7.01 & 8.29 & 9.51 \\
\hline \multirow[t]{2}{*}{11} & EWMA & 15 & 13 & 9 & 1.70 & 3.11 & 5.11 & 7.85 & 11.34 & 15.65 & 20.50 \\
\hline & CES & 20 & 18 & 14 & 2.38 & 3.35 & 5.04 & 6.68 & 8.60 & 10.99 & 13.83 \\
\hline $\mathrm{MA}(1)$ & IND & 117 & 78 & 120 & 2.52 & 2.93 & 5.13 & 5.49 & 7.04 & 7.45 & 8.92 \\
\hline \multirow[t]{2}{*}{12} & EWMA & 22 & 19 & 14 & 2.24 & 3.05 & 4.63 & 5.43 & 7.64 & 9.02 & 11.96 \\
\hline & CES & 29 & 26 & 20 & 2.44 & 2.91 & 4.99 & 5.45 & 7.32 & 8.01 & 10.07 \\
\hline
\end{tabular}


Consider, for example, $\operatorname{ARMA}(1,1)$ Model 1 and AR(1) Model 10 in Table 5. Both exhibit a similar level of sustained forecast recovery ( 0.50 versus 0.53 ). Model 10's forecast errors attain a sustained level of shift at $t=r+1$, while Model 1's forecast errors attain a sustained level at $t=r+34$. Although Model 10 has a slightly higher sustained level of forecast recovery, the Individuals control chart performs better when applied to Model 1 . The reason for this difference is a result of the magnitude of the gradually recovering forecast errors of Model 1. The Individuals chart takes advantage of the magnitude of forecast errors from time periods $t=r+1$ to $t=r+33$. Again, the $\mathrm{AR}(1)$ process forecast errors recover immediately to the sustained level of the shift at time period $t=$ $r+1$. The other control charts also exhibit similar behavior when applied to these two models.

Consider another example using Model 1 compared with MA(1) Model 12 in Table 5. Both exhibit similar levels of sustained forecast recovery $(0.50$ versus 0.53$)$, and both models attain a sustained level of shift at approximately $t$ $=r+34$. Both models exhibit gradually recovering forecast errors, but again the magnitude of the recovering forecast errors has a profound effect on the control charts. While the forecast errors arising from Model 1 gradually decrease from $\mathrm{E}\left(e_{t}^{*}\right)=$ 0.95 to 0.50 , those for Model 12 oscillate between values from $\mathrm{E}\left(e_{t}\right)=0.10$ to 0.91 until converging upon the sustained level of the shift at $\mathrm{E}\left(e_{t}^{*}\right)=$ 0.53 . As a result of this oscillating behavior, the control charts applied to Model 12 do not perform as well as the same charts applied to Model 1 even though Model 12 has a higher sustained level of the shift.

Many $\operatorname{ARMA}(1,1)$ processes exhibit oscillating behavior of forecast errors to some degree. The worst cases are those in which the forecast errors oscillate between values that alter in sign as well as magnitude and finally converge to the sustained level of the shift. $\operatorname{ARMA}(1,1)$ Models 3 and 4 in Table 5 are good examples of forecast errors exhibiting this oscillation behavior. Table 1 displays this behavior numerically for a shift of size $c=1 \sigma_{\varepsilon}$. The forecast errors in Model 3 oscillate between sequential values that differ in sign as well as absolute magnitude. The forecast errors in Model 4 oscillate between sequential values that differ in sign, but the absolute magnitudes of the forecast errors are very similar.

The behavior of the forecast errors in Model 4 dampens the performance of any control chart that requires the summing or averaging of forecast errors over time such as the EWMA or CUSUM control charts. If the forecast errors differ in sign but not in absolute magnitude, the result is a canceling-out effect of summed or averaged forecast errors, until finally reaching the sustained level of the shift. Models producing forecast errors that differ in sign as well as absolute magnitude (Model 3) experience the same canceling out effect but not to the same degree as is seen in Model 4.

Consider a comparison of the performance of control charts applied to Models 3 and 4. Both exhibit the same level of sustained forecast recovery (0.03). Model 3's forecast errors attain a sustained level of shift at $t=r+5$, while Model 4's forecast errors attain a sustained level at $t=r+$ 38. Longer time until sustained recovery is attained usually provides for an all around better chart performance for a given sustained level of a shift, but the oscillation behavior of the forecast errors in Model 4 negates this advantage in the case of the EWMA control chart. The Individuals control chart takes advantage of the magnitude of the recovering forecast errors in Model 4, ignoring the sign of each forecast error value. As a result, the Individuals chart applied in Model 4 was found to have phenomenally better ARL, MRL, and CDF performance than in the case of Model 3, over all shift sizes. In contrast, the EWMA control chart applied in Model 4 was found to perform significantly worse than in the case of Model 3 providing ARLs, MRLs, and CDFs that are lower for every shift size. Although the EWMA chart suffers in Model 4, the good performance of the Individuals chart results in CES control chart performance that is also good.

\section{Recommendations}

As a result of the phenomenon of forecast recovery and the behavior of recovering forecast errors, the authors have several recommendations in regards to selecting the appropriate control chart to use with various autocorrelated processes. The practitioner should: 
1. Determine the appropriate ARMA model and parameters regarding the process to be monitored, and use Eq. (9) to estimate the degree of forecast recovery.

2. Use Eq. (4) to determine the effect of forecast recovery on the forecast errors that will result from a step-shift of size $c$ in the mean of the underlying ARMA process.

3. Use one of Eq. (5), (6), or (7), depending on if the model is an $\operatorname{ARMA}(1,1), \operatorname{AR}(1)$, or MA(1), to determine the sustained level of recovery resulting from the step-shift of size $c$. The expected behavior of the recovering forecast errors should also be studied in regards to the rate of recovery, oscillation, the magnitude and sign of recovering forecast if oscillating, and the expected sampling period when the forecast will recover to the sustained level.

4. Select and apply the control chart who's performance is least affected by the forecast recovery, in face of the magnitude of the shift to be detected as well as the behavior of the recovering forecast.

The practitioner should take note that in the selection of the control chart, one first determines the magnitude of the shift that is deemed most important to detect. Recall, while the Individuals chart is best suited for rapidly detecting relatively large shifts, the EWMA chart is best suited for the eventual detection of small shifts. The CES chart serves as a compromise. Second, one must bear in mind that the behavior of recovering forecast might yield an otherwise favorable chart unsuitable for the monitoring the process at hand.

\section{Conclusion}

This article provided a description of various models for autocorrelated data, as well as an introduction to the Box-Jenkins OSA forecast and forecast error often used to monitor an autocorrelated process. Also provided was a mathematical description of the impact of forecast recovery on the $\operatorname{ARMA}(\mathrm{p}, \mathrm{q})$ process, and particularly the $\operatorname{ARMA}(1,1), \operatorname{AR}(1)$, and $\operatorname{MA}(1)$ processes.

Additionally, the article included a discussion concerning the relationship between initial/sustained rates of forecast recovery, and a model's particular parameter values and first order autocorrelation structure. It was shown that while the rates of forecast recovery differ for all models, these recovery rates are indeed a function of the model parameters. Additionally, knowledge of first order autocorrelation was shown helpful in determining the degree of sustained forecast error recovery in the $\operatorname{ARMA}(1,1), \operatorname{AR}(1)$, and $\operatorname{MA}(1)$ processes. Examples were given of various $\operatorname{ARMA}(p, q)$ forecast error recovery rates over time, while tables were provided relating the sustained expected value of forecast errors for a wide variety of $\operatorname{ARMA}(\mathrm{p}, \mathrm{q})$ processes.

Finally, it was found that the sustained level of forecast recovery following a shift had a tremendous effect on the performance of each control chart examined. The rate of recovery as well as the absolute magnitude and sign of forecast errors prior to attaining the sustained level of recovery were found to greatly influence the performance of the control charts. It was shown that for a given shift and sustained level of recovery, the control charts generally perform worse when applied to the forecast errors arising from AR(1) processes. The worsening of performance was shown to be due to the sudden forecast recovery characteristics inherent in these processes. As a result of the phenomenon of forecast recovery and the behavior of recovering forecasts, recommendations were made in regards to a practitioner selecting the most appropriate control chart for various ARMA processes.

\section{References}

Adams, B. M., Woodall, W. H., \& Superville, C. R. (1994). Discussion of run-length distributions of special-cause control charts for correlated processes by D. G. Wardell, H. Moskowitz, and R. D. Plante. Technometrics, 36, 19-21.

Alwan, L. C., \& Roberts, H. V. (1988). Time-series modeling for statistical process control. Journal of Business and Economic Statistics, 6, 87-95.

Atienza, O. O., Tang, L. L., \& Ang, B. W. (1998). A SPC procedure for detecting level shift of autocorrelated processes. Journal of Quality Technology, 30, 340-351. 
Box, G. E. P., Jenkins, G. M., \& Reinsel, G. C. (1994). Time series analysis, forecasting and control. ( $3^{\text {rd }}$. ed.). NJ: Prentice-Hall, Engelwood Cliffs.

Lin, W. S. W., \& Adams, B. M. (1996). Combined control charts for forecast-based monitoring schemes. Journal of Quality Technology, 28, 289-301.

Lu, C. W., \& Reynolds, M. R. Jr. (2001). CUSUM charts for monitoring an autocorrelated process. Journal of Quality Technology, 33, 316334.

Lu, C. W., \& Reynolds, M. R. Jr. (1999a). EWMA control charts for monitoring the mean of autocorrelated processes. Journal of Quality Technology, 31, 166-188.

Lu, C. W., \& Reynolds, M. R. Jr. (1999b). Control charts for monitoring the mean and variance of autocorrelated processes. Journal of Quality Technology, 31, 259-274.
Montgomery, D. C., \& Mastrangelo, C. M. (1991). Some statistical process control methods for autocorrelated data. Journal of Quality Technology, 23, 179-204.

Superville, C. R., \& Adams, B. M. (1994). An evaluation of forecast-based quality control schemes. Communications in Statistics: Simulation and Computation, 23, 645-661.

Vasilopoulos, A. V., \& Stoamboulis, A.P. (1978). Modification of control chart limits in the presence of data correlation. Journal of Quality Technology, 10, 20-30.

Wardell, D. G., Moskowitz, H. and Plante, R. D. (1994). Run-length distributions of specialcause control chartsfor correlated processes. Technometrics, 36, 3-16. 